Geometric concept of isokinetic constraint for a system of particles

\author{
Martin Swaczyna and Petr Volný
}




\title{
GEOMETRIC CONCEPT OF ISOKINETIC CONSTRAINT FOR A SYSTEM OF PARTICLES
}

\author{
MARTIN SWACZYNA AND PETR VOLNÝ
}

\begin{abstract}
The paper deals with the geometric concept of mechanical systems of $N$ particles. The systems are modelled on the Cartesian product $\mathbb{R} \times \mathbb{X}^{N}$ and its first jet prolongation $J^{1}(\mathbb{R} \times$ $\left.\mathbb{X}^{N}\right)=\mathbb{R} \times T \mathbb{X}^{N}$, where $\mathbb{X}$ is a 3 -dimensional Riemannian manifold with a metric $G$. The kinetic energy $T$ of the system of $N$-particles is interpreted by means of the weighted quadratic form $\bar{Q}_{\mathscr{E}}$ associated with the weighted metric tensor $\mathcal{G}$ which arises from the original metric tensor $G$ and the system of $N$ particles $m_{1}, \ldots, m_{N}$. A requirement for the kinetic energy of the system of $N$ particles to be constant is regarded as a nonholonomic, so-called isokinetic constraint and it is defined as a fibered submanifold $\mathbb{T}$ of the jet space $\mathbb{R} \times T \mathbb{X}^{N}$ endowed with a certain distribution $\mathcal{C}$ called canonical distribution, which has the meaning of generalized admissible displacements of the system of particles subject to the isokinetic constraint. Vector generators of the canonical distribution are found.
\end{abstract}

2000 Mathematics Subject Classification: 37J60, 70F25, 70G10, 70G45

Keywords: mechanical systems of particles, kinetic energy, metric tensor, nonholonomic constraints, isokinetic constraints, isokinetic canonical distribution

\section{INTRODUCTION}

Our motivation for the study of systems of particles whose movement is restricted by the a priori requirement that kinetic energy of the system remains constant comes from our previous investigations of a simple mechanical system of one particle moving with constant speed in the homogeneous gravitational field [7] and in the central gravitational field inspired by [3]. This theoretical problem of classical mechanics has the origin at the end of the 17th century (Jacob Bernoulli, Pierre Varignon). However, for a full understanding of all aspects of the problem, it was necessary to correctly interpret the requirement of constant instantaneous speed of a particle as a nonlinear nonholonomic constraint and to apply modern geometric approach based on the concept of nonholonomic constraints as a certain submanifold in the "velocity" space.

The condition preserving constant kinetic energy of systems of particles (isokinetic constraint) arose as a natural generalization of the condition of constant speed of one particle (isotachytonic constraint). For further study of these "isokinetically" 
constrained systems, it is necessary to propose a suitable approach to isokinetic constraint. First, it was the need to precisely analyze the concept of kinetic energy of systems of particles, to find its geometric essence, and then to interpret the isokinetic constraint as the corresponding submanifold in the corresponding evolution space and, finally, to adopt general geometric theory of nonholonomic constraints [1,2] and [4-6]. In the future, we will try to apply this general concept to a non-trivial example, so-called "isokinetic two body problem", where speeds of two gravitationally interacting particles are not constant but the total kinetic energy of this system remains constant.

\section{GeOMETRIC MOdEl OF THE SYSTEM OF $N$ PARTICleS}

A typical configuration space for the system of one particle in classical mechanics is the Euclidean space $\mathbb{E}_{3}$ or, in general, some 3-dimensional smooth manifold $\mathbb{X}$. A suitable geometric structure for modelling motions of the particle is the fibered manifold $\pi: \mathbb{R} \times \mathbb{X} \rightarrow \mathbb{R}$ and its first jet prolongation $\pi_{1}: J^{1}(\mathbb{R} \times \mathbb{X})=\mathbb{R} \times T \mathbb{X} \rightarrow \mathbb{R}$, $T \mathbb{X}$ is the tangent space to $\mathbb{X}$. The spaces $\mathbb{R} \times \mathbb{X}, T \mathbb{X}, \mathbb{R} \times T \mathbb{X}$ will be called space of events, phase space and evolution space for the system of one particle, respectively. Let us denote by $\left(U_{\kappa}, \varphi_{\kappa}\right)$ some local chart on $\mathbb{X}, U_{\kappa} \subset \mathbb{X}$ is an open subset and $\varphi_{\kappa}=\left(q^{1}, q^{2}, q^{3}\right)$ are called generalized curvilinear local coordinates on $\mathbb{X}$. There naturally arise the following local coordinates: $\left(t, q^{S}\right)$ on $\mathbb{R} \times \mathbb{X},\left(q^{S}, \dot{q}^{s}\right)$ on $T \mathbb{X}$, and $\left(t, q^{s}, \dot{q}^{s}\right)$ on $\mathbb{R} \times T \mathbb{X}, 1 \leq s \leq 3 ; t$ is a global coordinate on $\mathbb{R}$ (time axis).

Let us consider a system of $N$ particles. The set of all possible configurations of this system, configuration space $Y$, will be treated in "separable" form of the Cartesian product $\mathbb{Y}=\mathbb{X} \times \mathbb{X} \times \cdots \times \mathbb{X}=\mathbb{X}^{N}$, where each $\mathbb{X}$ represents the configuration space of a single particle, $\operatorname{dim} \mathbb{X}=3$. We use the notation ${ }^{i} \mathbb{X}$ for the configuration space of the $i$-th particle. There arise naturally the projections $\operatorname{pr}_{i}: Y \rightarrow{ }^{i} \mathbb{X}$, $\operatorname{pr}_{i}(Y)={ }^{i} X, Y$ is instantaneous configuration of the system and ${ }^{i} X$ is the corresponding position of the $i$-th particle. The configuration space $Y=\mathbb{X}^{N}$ has the structure of the smooth manifold given by the atlas $A t \mathrm{Y}=\left\{\left(U_{\kappa_{1} \ldots \kappa_{N}}, \varphi_{\kappa_{1} \ldots \kappa_{N}}\right)\right\}=$ $\left\{\left({ }^{1} U_{\kappa_{1}} \times \cdots \times{ }^{N} U_{\kappa_{N}},{ }^{1} \varphi_{\kappa_{1}} \times \cdots \times \times{ }^{N} \varphi_{\kappa_{N}}\right)\right\}$, where $\left({ }^{i} U_{\kappa_{i}},{ }^{i} \varphi_{\kappa_{i}}\right),{ }^{i} \varphi_{\kappa_{i}}=\left({ }^{i} q^{1},{ }^{i} q^{2},{ }^{i} q^{3}\right)$ is some coordinate system on ${ }^{i} \mathbb{X}$ for each $i, 1 \leq i \leq N$.

Thus, the instantaneous position $Y$ of the system is described by the $3 N$-tuple $\left(q^{1}(Y), q^{2}(Y), \ldots, q^{3 N}(Y)\right)$, such that every triple $\left(q^{3 i-2}(Y), q^{3 i-1}(Y), q^{3 i}(Y)\right)=$ $\left({ }^{i} q^{1}\left({ }^{i} X\right),{ }^{i} q^{2}\left({ }^{i} X\right),{ }^{i} q^{3}\left({ }^{i} X\right)\right)$ relates to the position ${ }^{i} X$ of the $i$-th particle.

Let $I \subset \mathbb{R}$ be such a time interval during which there is no collision of particles. By a regular motion of the system of $N$ particles (motion without collisions) in time interval $I$ we mean the twice differentiable mapping $c: I \ni t \mapsto c(t) \in \mathbb{X}^{N}$. Hence, $c$ is the curve on $\mathbb{X}^{N}$. Especially, $\mathrm{pr}_{i} \circ c={ }^{i} c: I \rightarrow{ }^{i} \mathbb{X}$ represents the regular motion of the $i$-th particle, it is a curve in ${ }^{i} \mathbb{X}$.

The space $\mathbb{R} \times \mathbb{X}^{N}$ and $\mathbb{R} \times{ }^{i} \mathbb{X}$ is called the space of events of the system of $N$ particles and the space of events of the $i$-th particle, respectively. The spaces of events 
together with the projections $\mathrm{pr}_{0}=\pi: \mathbb{R} \times \mathbb{X}^{N} \rightarrow \mathbb{R},{ }^{i} \mathrm{pr}_{0}={ }^{i} \pi: \mathbb{R} \times{ }^{i} \mathbb{X} \rightarrow \mathbb{R}$ have the structure of the fibered manifolds over 1-dimensional base $\mathbb{R}$; it is a basic underlying structure for modeling motions of particles without collisions. Fibers $\pi^{-1}(t)$ and ${ }^{i} \pi^{-1}(t)$ at any time $t$ are just the configuration spaces $\mathbb{X}^{N}$ and ${ }^{i} \mathbb{X}$.

Let $Y \in \mathbb{X}^{N}, Y=\left({ }^{1} X, \ldots,{ }^{N} X\right)$ be some configuration of the mechanical system of $N$ particles. The tangent vector space $T_{Y} \mathbb{X}^{N}$ to the configuration space $\mathbb{X}^{N}$ at the configuration $Y$ and the tangent vector spaces $T_{i}{ }_{X}{ }^{i} \mathbb{X}$ to the configuration spaces ${ }^{i} \mathbb{X}$ of single particles at the positions ${ }^{i} X$ have the meaning of the space of velocities of the system of $N$ particles at the configuration $Y$ and spaces of velocities of single particles at the corresponding positions ${ }^{i} X$. Since $T_{Y} \mathbb{X}^{N}=T_{1}{ }^{1} \mathbb{X} \times \cdots \times T_{N}{ }^{N} \mathbb{X}$, every velocity vector $\boldsymbol{v} \in T_{Y} \mathbb{X}^{N}$ consists of the velocity vectors ${ }^{i} \boldsymbol{v}$ of the single particles, i.e. $\boldsymbol{v}=\left({ }^{1} \boldsymbol{v}, \ldots,{ }^{N} \boldsymbol{v}\right),{ }^{i} \boldsymbol{v} \in T_{i}{ }^{i} \mathbb{X}$. In some coordinate system $(U, \varphi) \in$ At $\mathrm{Y}, U=U_{\kappa_{1} \ldots \kappa_{N}} \subset \mathrm{Y}, \varphi=\varphi_{\kappa_{1} \ldots \kappa_{N}}=\left({ }^{i} q^{s}\right), 1 \leq i \leq N, 1 \leq s \leq 3$, $\boldsymbol{v}={ }^{i} v^{s}\left(\partial / \partial^{i} q^{s}\right)_{Y}={ }^{i} \dot{q}^{s}(\boldsymbol{v})\left(\partial / \partial^{i} q^{s}\right)_{Y}$, where ${ }^{i} v^{s}={ }^{i} \dot{q}^{s}(\boldsymbol{v})$ are components of $\boldsymbol{v}=\left({ }^{i} \boldsymbol{v}\right)=\left({ }^{1} \boldsymbol{v}, \ldots,{ }^{N} \boldsymbol{v}\right)$, and where every triple $\left({ }^{i} v^{1},{ }^{i} v^{2},{ }^{i} v^{3}\right)=\left({ }^{i} \dot{q}^{1},{ }^{i} \dot{q}^{2},{ }^{i} \dot{q}^{3}\right)$ represents components of the velocity vector ${ }^{i} \boldsymbol{v}$ of the $i$-th particle with respect to the basis $\left\{\left(\partial / \partial^{i} q^{s}\right)_{X_{i}}\right\}$ of the space $T_{i}{ }^{i} \mathbb{X}$.

Put $T \mathbb{X}^{N}=\cup_{Y \in \mathbb{X}^{N}} T_{Y} \mathbb{X}^{N}$ and $T U=\cup_{Y \in U} T_{Y} \mathbb{X}^{N}$. Denote $\tau: T \mathbb{X}^{N} \rightarrow \mathbb{X}^{N}$ a surjective mapping defined as $\boldsymbol{v} \in T \mathbb{X}^{N} \mapsto \tau(\boldsymbol{v})=Y$ such that $\boldsymbol{v} \in T_{Y} \mathbb{X}^{N}$. Quite analogously, for the $i$-th particle, $\tau_{i}: T^{i} \mathbb{X} \rightarrow{ }^{i} \mathbb{X}, \tau_{i}\left({ }^{i} \boldsymbol{v}\right)={ }^{i} X,{ }^{i} \boldsymbol{v} \in T_{i} X{ }^{i} \mathbb{X}$. The set $T \mathbb{X}^{N}$ has the structure of a smooth manifold of dimension $6 N$ such that, for every coordinate system $(U, \varphi), \varphi=\left({ }^{i} q^{s}\right)$, on $\mathbb{X}^{N}$ is $(T U, T \varphi), T \varphi=\left({ }^{i} q^{s},{ }^{i} \dot{q}^{s}\right)$, coordinate system on $T \mathbb{X}^{N}$. The manifold $T \mathbb{X}^{N}$ is called tangent fibered space of the configuration manifold $\mathbb{X}^{N}$ and plays the role of the phase space, space of all possible configurations and of all possible velocities of the mechanical system of $N$ particles. Similarly, the manifolds $T^{i} \mathbb{X}$ have the meaning of phase spaces for single particles.

The first jet prolongation $J^{1}\left(\mathbb{R} \times \mathbb{X}^{N}\right)$ of the space of events $\mathbb{R} \times \mathbb{X}^{N}$ is the manifold $\mathbb{R} \times T \mathbb{X}^{N}$ called evolution space of the system of $N$ particles. Associated coordinates on $\mathbb{R} \times T \mathbb{X}^{N}$ are denoted by $\left(t,{ }^{i} q^{s},{ }^{i} \dot{q}^{s}\right)$. There naturally arise the projections $\pi_{1}$ : $\mathbb{R} \times T \mathbb{X}^{N} \rightarrow \mathbb{R}$, and $\pi_{1,0}: \mathbb{R} \times T \mathbb{X}^{N} \rightarrow \mathbb{R} \times \mathbb{X}^{N}, \pi_{1,0}=\operatorname{id}_{\mathbb{R}} \times \tau$. The evolution space and the corresponding projections can be also distinguished for the single particles, then, upright index $i$ is used.

\section{GEOMETRIC APPROACH TO THE KINETIC ENERGY}

Suppose that there exists on each factor $\mathbb{X}$ a Riemannian metric tensor $G$, a symmetric bilinear non-degenerate form $G_{X}: T_{X} \mathbb{X} \times T_{X} \mathbb{X} \rightarrow \mathbb{R}$, which is at each point $X \in \mathbb{X}$ positive definite, i.e., the inner product between tangent vectors. We use the notation ${ }^{i} G$ for the specification of the metric tensor $G$ on the factor ${ }^{i} \mathbb{X}$. Coordinate representation of the metric tensor ${ }^{i} G$ in the chart $\left(T^{i} U_{\kappa_{i}}, T^{i} \varphi_{\kappa_{i}}\right)$ on $T^{i} \mathbb{X}$ associated with the chart $\left({ }^{i} U_{\kappa_{i}},{ }^{i} \varphi_{\kappa_{i}}\right),{ }^{i} \varphi_{\kappa_{i}}=\left({ }^{i} q^{s}\right)$ on $\mathbb{X}$ is ${ }^{i} G_{X_{i}}\left({ }^{i} \boldsymbol{u},{ }^{i} \boldsymbol{v}\right)=$ 
${ }^{i} G_{k l}{ }^{i} \dot{q}^{k}\left({ }^{i} \boldsymbol{u}\right){ }^{i} \dot{q}^{l}\left({ }^{i} \boldsymbol{v}\right)={ }^{i} G_{k l}{ }^{i} \dot{q}^{k i} \dot{q}^{l},{ }^{i} \boldsymbol{u},{ }^{i} \boldsymbol{v} \in T_{X_{i}}{ }^{i} \mathbb{X}$. The associated quadratic form $Q^{{ } G}$ will be denoted by ${ }^{i} Q$. By Sylvester's law of inertia, there exists a basis of the tangent space $T_{i} X{ }^{i} \mathbb{X}$ and a local chart $\left({ }^{i} U_{0},{ }^{i} \varphi_{0}\right) \in A t^{i} \mathbb{X},{ }^{i} X \in{ }^{i} U_{0},{ }^{i} \varphi_{0}=\left({ }^{i} w^{s}\right)$ so that the quadratic form ${ }^{i} Q$ becomes diagonal

$$
{ }^{i} Q=\delta_{k l}{ }^{i} \dot{w}^{k}{ }^{i} \dot{w}^{l}=\left({ }^{i} \dot{w}^{1}\right)^{2}+\left({ }^{i} \dot{w}^{2}\right)^{2}+\left({ }^{i} \dot{w}^{3}\right)^{2} .
$$

On the configuration space $\mathbb{X}^{N}$, there naturally arises a metric tensor $\mathscr{E}=\sum_{i=1}^{N}{ }^{i} G$ and the associated quadratic form $Q_{e}=\sum_{i=1}^{N}{ }^{i} Q$, more precisely,

$$
\mathscr{E}(\boldsymbol{u}, \boldsymbol{v})=\sum_{i=1}^{N} G\left({ }^{i} \boldsymbol{u},{ }^{i} \boldsymbol{v}\right), \quad Q_{\mathscr{E}}(\boldsymbol{v}, \boldsymbol{v})=\sum_{i=1}^{N} Q\left({ }^{i} \boldsymbol{v},{ }^{i} \boldsymbol{v}\right) .
$$

Another metric tensor and corresponding quadratic form on $\mathbb{X}^{N}$ directly associated with the system of $N$ particles with masses $m_{1}, \ldots, m_{N}$ are

$$
\overline{\mathscr{g}}=\frac{\sum_{i=1}^{N} m_{i}{ }^{i} G}{\sum_{i=1}^{N} m_{i}}, \quad \bar{Q}_{\overline{\mathscr{g}}}=\frac{\sum_{i=1}^{N} m_{i}{ }^{i} Q}{\sum_{i=1}^{N} m_{i}},
$$

the so-called weighted metric tensor and weighted quadratic form associated with the system of $N$ particles considered.

Let us denote by $M=\sum_{i=1}^{N} m_{i}$ the total mass of the system. The total kinetic energy of the system of $N$ particles with masses $m_{1}, \ldots, m_{N}$ can be interpreted by means of

$$
T=\frac{1}{2} M \bar{Q}_{\overline{\mathscr{G}}}(\boldsymbol{v})=\frac{1}{2} M \overline{\mathscr{G}}(\boldsymbol{v}, \boldsymbol{v})=\frac{1}{2} \sum_{i=1}^{N} m_{i}{ }^{i} Q\left({ }^{i} \boldsymbol{v}\right)=\frac{1}{2} \sum_{i=1}^{N} m_{i}{ }^{i} G\left({ }^{i} \boldsymbol{v},{ }^{i} \boldsymbol{v}\right),
$$

or in some coordinates on $T \mathbb{X}^{N}$

$$
T=\sum_{i=1}^{N} \frac{1}{2} m_{i}^{i} G_{k l}{ }^{i} \dot{q}^{k}{ }^{i} \dot{q}^{l}
$$

Quite analogously, one can introduce the total kinetic energy $T^{m_{j_{1}}, \ldots, m_{j_{K}}}$ of any subsystem of $K$ particles $m_{j_{1}}, \ldots, m_{j_{K}}$ of the system $m_{1}, \ldots, m_{N}$ by means of the corresponding weighted quadratic form associated with the subsystem considered.

\section{GEOMETRIC CONCEPT OF ISOKINETIC CONSTRAINTS}

\subsection{Nonholonomic constraints}

By constraints we mean conditions in the form of equations which restrict the possible configurations and velocities of mechanical systems of $N$ particles. By nonholonomic constraints we mean a system of non-integrable differential equations defined on the evolution space $\mathbb{R} \times T \mathbb{X}^{N}$. 
From the geometric point of view, $p$ nonholonomic constraints generate certain submanifold, called constraint submanifold $\mathbb{W} \subset \mathbb{R} \times T \mathbb{X}^{N}$, dim $\mathbb{N}=6 N+1-p$, which is fibered over $\mathbb{R} \times \mathbb{X}^{N}$, and also over $\mathbb{R}$, i.e., there arises the fibered manifold $\bar{\pi}_{1,0}: \mathbb{H} \rightarrow \mathbb{R} \times \mathbb{X}^{N}$, where $\bar{\pi}_{1,0}$ is the restriction of the projection $\pi_{1,0}: \mathbb{R} \times$ $T \mathbb{X}^{N} \rightarrow \mathbb{R} \times \mathbb{X}^{N}$ onto $\mathbb{W}$, and the fibered manifold $\bar{\pi}_{1}: \mathbb{H} \rightarrow \mathbb{R}$ where $\bar{\pi}_{1}=$ $\left.\pi_{1}\right|_{\mathbb{H}}$. We denote by $\iota: \mathbb{H} \rightarrow \mathbb{R} \times T \mathbb{X}^{N}$ the canonical embedding. Especially, constraint submanifold of the form $\mathbb{H}=\mathbb{R} \times \mathbb{H}_{0}$, where $\mathbb{H}_{0} \subset T \mathbb{X}^{N}$ represents time independent nonholonomic constraints.

Let $\mathbb{W}$ be the constraint submanifold $\mathbb{W} \subset \mathbb{R} \times T \mathbb{X}^{N}$. At each point $H \in \mathbb{W}$, there exists a local chart $(V, \chi)$ on $\mathbb{R} \times T \mathbb{X}^{N}, V=I \times T U, \chi=\operatorname{id}_{I} \times T \varphi$, where $I \subset \mathbb{R}$ is a time interval and $(T U, T \varphi)$ is some coordinate system on $T \mathbb{X}^{N}, T \varphi=\left({ }^{i} q^{s},{ }^{i} \dot{q}^{s}\right)$, $1 \leq i \leq N, 1 \leq s \leq 3$, such that on $\mathbb{H}_{V}=\mathbb{W} \cap V$ the submanifold $\mathbb{R}$ is locally expressed by the equations

$$
f^{a}\left(t,{ }^{i} q^{s}, \dot{q}^{s}\right)=0, \quad 1 \leq a \leq p,
$$

where $\operatorname{rank}\left(\frac{\partial f^{a}}{\partial^{i} \dot{q}^{s}}\right)=p$.

The equations can be locally solved with respect to some selected $p$ coordinates ${ }^{i} \dot{q}^{s}$. Assume that $p<N$, then, without loss of generality, one can assort some $p$ particles $\left\{m_{i_{a}}\right\}, i_{a} \in\{1,2, \ldots, N\}, 1 \leq a \leq p$, and, for each particle $m_{i_{a}}$, one can choose some $s_{i_{a}}$ component of its velocity ${ }^{i_{a}} \boldsymbol{v}, s_{i_{a}} \in\{1,2,3\}$. Hence, we obtain equations of constraints in normal (explicit) form

$$
{ }^{i_{a}} \dot{q}^{s_{i a}}=g^{a}\left(t,{ }^{i} q^{s},{ }^{j} \dot{q}^{s},{ }^{i_{a}} \dot{q}^{\bar{s}_{i a}}\right),
$$

with $1 \leq j \leq N, j \neq i_{a}, 1 \leq \bar{s}_{i_{a}} \leq 3, \bar{s}_{i_{a}} \neq s_{i_{a}}$, where ${ }^{i_{a}} \dot{q}^{s_{i}}$ are expressed as a functions $g^{a}$ of remaining coordinates.

\subsection{Isokinetic constraint submanifold}

Let us consider a situation such that the movement of the system of $N$ particles is restricted by the a priori condition that the total kinetic energy $T$ of the system remains constant and it equals to nonzero initial total kinetic energy $T_{0}$ of the system given by initial positions and initial velocities of single particles.

In some local chart $(V, \chi)$ on $\mathbb{R} \times T \mathbb{X}^{N}, V=I \times T U, I \subset \mathbb{R}$ a time interval, $\chi=$ $\operatorname{id}_{I} \times T \varphi$, where $(T U, T \varphi)$ is some coordinate system on $T \mathbb{X}^{N}, T \varphi=\left({ }^{i} q^{s}, i_{q^{s}}\right)$, $1 \leq i \leq N, 1 \leq s \leq 3$, the requirement for constant total kinetic energy of the system has the form

$$
T^{m_{1}, \ldots, m_{N}}=T=\sum_{i=1}^{N} \frac{1}{2} m_{i}{ }^{i} G_{k l}\left({ }^{i} q^{1},{ }^{i} q^{2},{ }^{i} q^{3}\right)^{i} \dot{q}^{k i} \dot{q}^{l}=T_{0}=\text { const. }
$$


This condition represents one nonholonomic constraint and generates a constraint submanifold $\mathbb{T} \subset \mathbb{R} \times T \mathbb{X}^{N}$ of the dimension $6 N$, called isokinetic constraint submanifold. Since $\mathbb{T}=\mathbb{R} \times \mathbb{T}_{0}$, where $\mathbb{T}_{0} \subset T \mathbb{X}^{N}$, it is time-independent nonholonomic constraint. Equation (4.3) is then local expression of the submanifold $\mathbb{T}_{V}=\mathbb{T} \cap V$ or $\mathbb{T}_{0 U}=\mathbb{T}_{0} \cap T U$. The rank condition (4.1) is evidently satisfied,

$$
\operatorname{rank}\left(\frac{\partial f}{\partial^{i} \dot{q}^{l}}\right)=\operatorname{rank}\left(m_{i}{ }^{i} G_{k l}{ }^{i} \dot{q}^{k}\right)=1,
$$

since the metric tensors ${ }^{i} G$ are positive and the total kinetic energy remains constant and nonzero, thus, the system cannot stop.

In order to obtain the normal form of the equation of isokinetic constraint submanifold $\mathbb{T}$ we will suppose that there exist some time intervals during which some particle is located just in such a coordinate chart on $\mathbb{X}$ in which the corresponding particular quadratic form $Q$ has the diagonal form (3.1). Without loss of generality, let us assume that, for instance, in time interval $I_{J}, J$-th particle is located in coordinate chart $\left({ }^{J} U_{0},{ }^{J} \varphi_{0}\right),{ }^{J} \varphi_{0}=\left({ }^{i} w^{s}\right)$ on ${ }^{i} \mathbb{X}$. Denote by $(W, \psi)$ the chart on $\mathbb{R} \times T \mathbb{X}^{N}, W=I_{J} \times T \bar{U}, \psi=\mathrm{id}_{I_{J}} \times T \bar{\varphi}$, where $(T \bar{U}, T \bar{\varphi})$ is such a coordinate system on $T \mathbb{X}^{N}$ that its $J$-th component is just the chart $\left({ }^{J} U_{0},{ }^{J} \varphi_{0}\right)$. Hence, the segment $\mathbb{T}_{W}=\mathbb{T} \cap W$ of the isokinetic submanifold $\mathbb{T}$ is locally expressed by the equation

$$
\sum_{i=1, i \neq J}^{N} \frac{1}{2} m_{i}{ }^{i} G_{k l}{ }^{i} \dot{q}^{k}{ }^{i} \dot{q}^{l}+\frac{1}{2} m_{J}\left(\left({ }^{J} \dot{w}^{1}\right)^{2}+\left({ }^{J} \dot{w}^{2}\right)^{2}+\left({ }^{J} \dot{w}^{3}\right)^{2}\right)=T_{0} .
$$

Finally, for instance, ${ }^{J} \dot{w}^{3}$ can be expressed explicitly

$$
\begin{aligned}
{ }^{J} \dot{w}^{3} & =g_{ \pm} \\
& = \pm \frac{1}{\sqrt{m_{J}}} \sqrt{2 T_{0}-\sum_{\substack{i=1 \\
i \neq J}}^{N} m_{i}{ }^{i} G_{k l}{ }^{i} \dot{q}^{k i} \dot{q}^{l}-m_{J}\left({ }^{J} \dot{w}^{1}\right)^{2}-m_{J}\left({ }^{J} \dot{w}^{2}\right)^{2}}
\end{aligned}
$$

thus the local isokinetic constraint $\mathbb{T}_{W}$ splits into two components $\mathbb{T}_{W}^{+}\left({ }^{J} \dot{w}^{3}>0\right)$ and $\mathbb{T}_{W}^{-}\left({ }^{J} \dot{w}^{3}<0\right)$.

\subsection{Isokinetic canonical distribution}

A fundamental object in the geometry of nonholonomic constraints is the canonical distribution discovered in [4], naturally associated with the constraint submanifold $\mathbb{H}$. It is a vector distribution $C \subset T \mathbb{W}$ of the dimension $6 N-2 p+1$ on the constraint submanifold $\mathbb{t}$ related to $p$ nonholonomic constraints (4.1). This distribution has the meaning of generalized possible displacements of mechanical systems constrained to $\mathbb{W}$. Particularly, its $\pi_{1}$-vertical subdistribution represents the space of 
$\mathbb{H}$-admissible virtual displacements and its $\pi_{1,0}$-vertical subdistribution represents the space of $\mathbb{-}$-admissible virtual velocities. The generators of the canonical distribution $\mathcal{C}$ for the case of general constraint submanifold are calculated in [4]. These formulas contain the equations of constraints in the normal form (4.2). The canonical distribution associated with the isokinetic constraint (4.3) is called isokinetic canonical distribution and denoted by $\ell_{\mathbb{T}}$, evidently $\ell_{\mathbb{T}} \subset T \pi$.

If we take into account equation (4.6) of local isokinetic submanifold $\mathbb{T}_{W}$ or more concretely, its "positive" component $\mathbb{T}_{W}^{+}\left({ }^{J} \dot{w}^{3}=g_{+}\right)$, we obtain the following vector generators of the local isokinetic canonical distribution $\ell_{\mathrm{T}_{W}^{+}}$

$$
\begin{aligned}
\xi_{0} & =\frac{\partial}{\partial t}+\frac{2 T_{0}}{m_{J} g_{+}} \frac{\partial}{\partial{ }^{J} w^{3}}, \\
i_{\xi_{r}} & =\frac{\partial}{\partial^{i} q^{r}}-\frac{m_{i}}{m_{J} g_{+}}{ }^{i} G_{r s}{ }^{i} \dot{q}^{s} \frac{\partial}{\partial^{J} w^{3}}, \\
J_{\xi_{1}} & =\frac{\partial}{\partial^{J} w^{1}}-\frac{{ }^{J} \dot{w}^{1}}{g_{+}} \frac{\partial}{\partial{ }^{J} w^{3}}, \\
J_{\xi_{2}} & =\frac{\partial}{\partial^{J} w^{2}}-\frac{{ }^{J} \dot{w}^{2}}{g_{+}} \frac{\partial}{\partial^{J} w^{3}}, \\
{ }^{i} \tilde{\xi}_{r} & =\frac{\partial}{\partial^{i} \dot{q}^{r}}, \\
J \tilde{\xi}_{1} & =\frac{\partial}{\partial^{J} \dot{w}^{1}}, \\
J \tilde{\xi}_{2} & =\frac{\partial}{\partial^{J} \dot{w}^{2}},
\end{aligned}
$$

where $1 \leq i \leq N, i \neq J, 1 \leq r, s \leq 3$. Note that the rank of every local isokinetic canonical distribution $\ell_{\mathbb{T}_{W}}$ is equal to $6 N-1$. The meaning of generators is the following: $\xi_{0}$ represents isokinetically admissible time translation of the system, ${ }^{i} \xi_{r}$ have the meaning of components of isokinetically admissible virtual displacements of single particles (except the $J$ th particle), ${ }^{J} \xi_{1},{ }^{J} \xi_{2}$ are the components of isokinetically admissible virtual displacements of the $J$ th particle, ${ }^{i} \tilde{\xi}_{r}$ play the role of the components of isokinetically admissible virtual velocities of single particles (except the $J$ th particle) and, finally, $J \tilde{\xi}_{1}, J \tilde{\xi}_{2}$ represent the components of isokinetically admissible virtual velocities of the $J$ th particle.

All the above considerations and calculations can also be applied in the case of the so-called partial isokinetic constraint, when the a priori condition keeps constant the total kinetic energy $T^{m_{j_{1}}, \ldots, m_{j_{K}}}=T_{0}^{m_{j_{1}}, \ldots, m_{j_{K}}}$ of any selected subsystem of $K$-particles $m_{j_{1}}, \ldots, m_{j_{K}}$. 


\section{ACKNOWLEDGEMENT}

The authors appreciate support of Grant GA 201/09/0981 of the Czech Science Foundations and of their departments.

\section{REFERENCES}

[1] V. I. Arnold, V. V. Kozlov, and A. I. Neishtadt, Mathematical Aspects of Classical and Celestial Mechanics. Berlin, Heidelberg: Springer Verlag, 2006.

[2] A. M. Bloch, Nonholonomic Mechanics and Control. New York: Springer Verlag, 2003.

[3] Y. F. Golubev, "Motion with a constant velocity modulus in a central gravitational field," J. Appl. Maths. Mechs., vol. 66, no. 6, pp. 1001-1013, 2002, English translation.

[4] O. Krupková, "Mechanical systems with nonholonomic constraints," J. Math. Phys., vol. 38, pp. 5098-5126, 1997.

[5] O. Krupková, "On the geometry of non-holonomic mechanical systems," in Proc. Conf. Diff. Geom. Appl., Brno, August 1998, O. Kowalski, I. Kolář, D. Krupka, and J. Slovák, Eds. Brno: Masaryk University, 1999, pp. 533-546.

[6] O. Krupková, "Recent results in the geometry of constrained systems," Rep. Math. Phys., vol. 49, pp. 269-278, 2002.

[7] M. Swaczyna and P. Volný, "Symmetries and conservation laws of uniform projectile motion," submitted.

Authors' addresses

Martin Swaczyna

University of Ostrava, Department of Mathematics, 30. dubna 22, 70103 Ostrava, Czech Republic

E-mail address: martin. swaczyna@osu.cz

Petr Volný

VŠB - Technical University of Ostrava, Department of Mathematics and Descriptive Geometry, 17. listopadu 15, 70833 Ostrava, Czech Republic

E-mail address: petr.volnyevsb.cz 